\title{
RADICALS AND BIMODULES
}

\author{
D. M. FOSTER
}

\begin{abstract}
In 1964, Andrunakievič and Rjabuhin showed that the general theory of radicals of associative rings may be presented in external form in the language of modules. In this paper, we show that this theory has a natural extension to varieties of algebras where, in this case, modules are replaced by bimodules. We close with some examples and a discussion of quadratic Jordan algebras.
\end{abstract}

1. Introduction and basics. In [2], Andrunakievič and Rjabuhin show that the general theory of radicals of associative rings may be presented in external form in the language of modules or, equivalently, representations. We will show that this theory has a natural extension to varieties of algebras where, in this case, modules (representations) are replaced by bimodules (birepresentations). Thus our results are analogous to those in [2], but do not reduce to them in the variety of associative algebras since we deal with bimodules.

We assume that the reader is familiar with the notions concerning varieties of algebras. Specifically, we assume a knowledge of bimodules and birepresentations. (For informations on varieties and the notation which we will adopt, see [5].) We also assume that the reader is familiar with the general theory of radicals of associative rings (for example, [3, Chapter 1]). We note that, since we have the fundamental homomorphism theorems available to us in arbitrary varieties, this general theory has a natural analogue in varieties.

Throughout this paper, $\Phi$ will be a fixed commutative, associative ring with 1, and the terms "module" and "algebra" will mean "unital left $\Phi$-module" and "unital left $\Phi$-algebra". The notation which we will adopt is standard.

2. General classes of $I$-bimodules in a variety $V(I)$. Unless otherwise stated, in this section, $V(I)$ will be a fixed but arbitrary variety of algebras.

Suppose $U \in V(I)$ and $M(U)$ is the multiplication algebra of $U$. If $x \in U$, write $\langle x\rangle=\{\alpha x+x T \mid \alpha \in \Phi, T \in M(U)\}$, the principal ideal of $U$ generated by $x$. If $x, y \in U$, then easily $\langle x-y\rangle \subseteq\langle x\rangle+\langle y\rangle$ and $\langle x y\rangle \subseteq$ $\langle x\rangle \cap\langle y\rangle$. Moreover, if $K \triangleleft U$ and $\varphi$ is the natural homomorphism from $U$

Received by the editors January 6, 1972 and, in revised form, April 22, 1972.

AMS (MOS) subject classifications (1970). Primary 17A99; Secondary 17C15.

Key words and phrases. Radical, bimodule, variety, quadratic Jordan algebra. 
onto $U / K$, then, for $x \in U, \varphi(\langle x\rangle)=\langle\varphi(x)\rangle$. Conversely, if $\bar{y} \in U / K$ and $y \in \varphi^{-1}(\bar{y})$, then $\varphi(\langle y\rangle)=\langle\bar{y}\rangle$.

Let $U \in V(I)$ and suppose $M$ is an $I$-bimodule for $U$. If $x \in U$, we set

$$
\begin{gathered}
M x=\{m x \mid m \in M\}, \quad M\langle x\rangle=\{m y \mid m \in M, y \in\langle x\rangle\}, \\
x M=\{x m \mid m \in M\}, \quad\langle x\rangle M=\{y m \mid m \in M, y \in\langle x\rangle\}, \\
{[0 \mid M]_{U}^{*}=\{x \in U \mid M x=x M=0\},} \\
{[0 \mid M]_{U}=\{x \in U \mid M\langle x\rangle=\langle x\rangle M=0\} .}
\end{gathered}
$$

Note that $[0 \mid M]_{U}^{*}$ consists of those $x \in U$ for which right and left multiplication by $x$ on $M$ is zero. Moreover, $[0 \mid M]_{U} \triangleleft U$, and $[0 \mid M]_{U}$ is the maximal ideal of $U$ which is contained in $[0 \mid M]_{U}^{*}$. In the event that, for all $x, y \in U$, right and left multiplication by $x y$ on $M$ can be written in terms of right and left multiplications by $x$ and $y$, then $[0 \mid M]_{U}=[0 \mid M]_{U}^{*}$. Of course, this is the case for alternative and Lie algebras. However, it is not the case for Jordan algebras. Indeed, Osborn [9] has given an example of a Jordan algebra $J$ and a $J$-bimodule $M$ for which $[0 \mid M]_{J}^{*}$ is not an ideal. We distinguish between $[0 \mid M]_{U}$ and $[0 \mid M]_{U}^{*}$ to underline our belief that both of them must be used if one tries to obtain structural information about algebras in $V(I)$ via bimodules (for example, see [9]).

Proposition 1. Suppose $U \in V(I), K \triangleleft U$, and $\bar{U}=U / K$. If $M$ together with the bilinear compositions $m(a+K)$ and $(a+K) m$ is an I-bimodule for $\bar{U}$, then, with the bilinear compositions $m a=m(a+K)$ and $a m=(a+K) m$, $M$ becomes an I-bimodule for $U$ such that $K \subseteq[0 \mid M]_{U}^{*}$. Conversely, if $M$ together with the bilinear compositions ma and am is an I-bimodule for $U$, and if $K \subseteq[0 \mid M]_{U}^{*}$, then, with the bilinear compositions $m(a+K)=m a$ and $(a+K) m=a m, M$ becomes an I-bimodule for $\bar{U}$ such that $[0 \mid M]_{\tilde{U}} \cong$ $\left([0 \mid M]_{U}\right) / K$. If $W \in V(I), W \cong U$, and $M$ is an I-bimodule for $U$, then $M$ may be regarded as an I-bimodule for $W$ and $[0 \mid M]_{U} \cong[0 \mid M]_{W}$.

Proof. The proof is an exercise in applying Theorem 9, p. 80 in [5], and the remarks at the beginning of the section.

Now suppose that for each $U \in V(I)$ there is assigned a (possibly empty) class $\Sigma_{U}$ of $I$-bimodules for $U$. Assume that if $W \in V(I)$ and $W \cong U$, then $\Sigma_{U}=\Sigma_{W}$. Let $\Sigma$ be the set of all $\Sigma_{U}$, and set, for each $U \in V(I), \operatorname{ker}(\Sigma, U)=\bigcap\left\{[0 \mid M]_{U} \mid M \in \Sigma_{U}\right\}$, where, if $\Sigma_{U}=\varnothing, \operatorname{ker}(\Sigma, U)=$ $U$. If $\operatorname{ker}(\Sigma, U)=U$ for $U \in V(I)$, we call $U \Sigma$-radical. If $\operatorname{ker}(\Sigma, U)=0$, we say that the class $\Sigma_{U}$ is faithful and that $U$ is $\Sigma$-semisimple.

Following Andrunakievič and Rjabuhin [2], we say $\Sigma$ is a general class of bimodules if the following axioms are satisfied.

(P.0) If $M \in \Sigma_{U}$, then $[0 \mid M]_{U}^{*} \neq U$. 
(P.1) If $K \triangleleft U, \bar{U}=U / K$, and $M \in \Sigma_{\bar{C}^{\prime}}$, then $M$, with the induced I-bimodule structure for $U$, lies in $\Sigma_{U}$.

(P.2) If $M \in \Sigma_{U}, K \triangleleft U, \bar{U}=U / K$, and $K \subseteq[0 \mid M]_{U}^{*}$, then $M$, with the induced $I$-bimodule structure for $\bar{U}$, lies in $\Sigma_{\bar{U}}$.

(P.3) If $\Sigma_{U}$ is faithful, then for each $0 \neq K \triangleleft U, \Sigma_{K} \neq \varnothing$.

(P.4) If, for each $0 \neq K \triangleleft U, \Sigma_{K} \neq \varnothing$, then $\Sigma_{U}$ is faithful.

Note that if $\Sigma$ satisfies (P.0)-(P.2), and if $K \triangleleft U \in V(I)$, then (P.1) and (P.2) just say that the admissible $I$-bimodules for $\bar{U}=U / K$ are precisely those $I$-bimodules for $U$ with the property that $K \subseteq[0 \mid M]_{U}^{*}$. Thus, from Proposition $1, \operatorname{ker}(\Sigma, U) / K=\operatorname{ker}(\Sigma, \bar{U})$, whence $U / \operatorname{ker}(\Sigma, U)$ is $\Sigma$-semisimple.

Lemma 1. Suppose the set $\Sigma$ has (P.0)-(P.2). Then, for each $U \in V(I)$, $\operatorname{ker}(\Sigma, U)$ coincides with $R(U)=\bigcap\{T \triangleleft U \mid U / T$ is $\Sigma$-semisimple $\}$.

Proof. Easily $R(U) \subseteq \operatorname{ker}(\Sigma, U)$. Conversely, suppose $T \triangleleft U$ and $\bar{U}=U / T$ is $\Sigma$-semisimple. Then $\operatorname{ker}(\Sigma, U) \subseteq \bigcap\left\{[0 \mid M]_{U} \mid M \in \Sigma_{U}, T \subseteq\right.$ $\left.[0 \mid M]_{U}\right\}=S$. Since $S / T=\operatorname{ker}(\Sigma, \bar{U})=0, S=T$. As $T$ was chosen arbitrarily, $\operatorname{ker}(\Sigma, U) \subseteq R(U)$, as desired.

THEOREM 1. Suppose the set $\Sigma$ has (P.0)-(P.3).

(1) The class $\mathscr{S}=\left\{U \in V(I) \mid \Sigma_{U}=\varnothing\right\}$ is a radical class.

(2) If $\mathscr{S}(U)$ denotes the $\mathscr{S}$-radical of $U$ for $U \in V(I)$, then $\mathscr{S}(U) \subseteq$ $\operatorname{ker}(\Sigma, U)$.

(3) (P.4) is satisfied iff for all $U \in V(I), \mathscr{S}(U)=\operatorname{ker}(\Sigma, U)$.

REMARK. We observe that (P.3) says that $U \Sigma$-semisimple implies $U$ is $\mathscr{S}$-semisimple, while (P.4) gives the converse (where $\mathscr{S}$-semisimple means no nonzero $\Sigma$-radical ideals).

Proof of Theorem 1. Suppose $L(\Sigma)=\left\{U \in V(I) \mid\right.$ there exists $M \in \Sigma_{U}$ such that $\left.[0 \mid M]_{U}=0\right\}$. Note that, by Proposition $1, L(\Sigma)$ is closed under isomorphisms. If $0 \neq B \triangleleft U \in L(\Sigma)$, by (P.3), $\Sigma_{B} \neq \varnothing$. Let $M \in \Sigma_{B}$ and $A=[0 \mid M]_{B}$. By (P.0), $A \neq B$ whence $\bar{B}=B \mid A \neq 0$ and $M \in \Sigma_{B}$. Since $[0 \mid M]_{B}=0, \bar{B} \in L(\Sigma)$. Thus every nonzero ideal of an algebra in $L(\Sigma)$ can be homomorphically mapped onto a nonzero algebra in $L(\Sigma)$ from which it follows that the class $\mathscr{S}^{*}=\{U \in V(I) \mid U$ cannot be homomorphically mapped onto a nonzero member of $L(\Sigma)\}$ is a radical class. Now if $\Sigma_{U} \neq \varnothing$ for $U \in V(I)$ and $M \in \Sigma_{U}$, then $0 \neq U /[0 \mid M]_{U} \in L(\Sigma)$. On the other hand, if $K \triangleleft U \in V(I)$ and $\bar{U}=U / K \in L(\Sigma)$, then there is an $M \in$ $\Sigma_{\tilde{U}}$ with $[0 \mid M]_{\tilde{U}}=0$. By (P.1), $M \in \Sigma_{U}$. From this we can conclude that $\mathscr{S}=\mathscr{S}^{*}$.

To prove (2), we recall that, for $U \in V(I), U / \operatorname{ker}(\Sigma, U)$ is $\Sigma$-semisimple, hence, by (P.3), $\mathscr{S}$-semisimple. Since $\mathscr{S}$ is a radical class, $\mathscr{S}(U)=$ $\bigcap\{T \triangleleft U \mid U / T$ is $\mathscr{S}$-semisimple $\} \subseteq \operatorname{ker}(\Sigma, U)$. 
Finally, suppose (P.4) is satisfied. Then $U / \mathscr{S}(U)$ being $\mathscr{S}$-semisimple implies $U / \mathscr{S}(U)$ is $\Sigma$-semisimple. So $\operatorname{ker}(\Sigma, U) \subseteq \mathscr{S}(U)$ by Lemma 1 . Conversely, suppose $\operatorname{ker}(\Sigma, U)=\mathscr{S}(U)$ and that $U$ is $\mathscr{S}$-semisimple. Then $0=\mathscr{S}(U)=\operatorname{ker}(\Sigma, U)$, i.e. $U$ is $\Sigma$-semisimple.

REMARK. The proof of (1) in the above theorem is patterned after Hentzel's proof in the associative case [4].

Next we show that if we impose an additional condition on $V(I)$, then we can prove the converse of Theorem 1 . Thus we say that $V(I)$ has $(*)$ if, for every $U \in V(I), U^{*}=\Phi 1 \oplus U \in V(I)$, i.e. the algebra obtained from $U$ by adjoining an identity element lies in $V(I)$. This assumption on $V(I)$ permits us to regard $U^{*} / T$ as an $I$-bimodule for $U$ for every $T \triangleleft U$ and, moreover, $\left[0 \mid U^{*} / T\right]_{U}=T$. We remark that if $I$ satisfies conditions $(\mathrm{H})$, $(\mathrm{U})$, and $(\mathrm{L})$ in the notation of [5] (see [5, Chapter 1, §6]), then $V(I)$ has $(*)$.

The idea now behind the next theorem is quite simple. If $\mathscr{P}$ is a radical property for $V(I)$, then, for $U \in V(I), \mathscr{P}(U)=\bigcap\{T \triangleleft U \mid \mathscr{P}(U / T)=0\}$. This, together with the assumption that $V(I)$ has $(*)$ will allow us to characterize $\mathscr{P}(U)$ as the intersection of the "annihilators" of certain bimodules.

THEOREM 2. Suppose $V(I)$ has $(*)$. If $\mathscr{P}$ is any radical property for $V(I)$, then there exists, for each $U \in V(I)$, a set $\Sigma_{U}$ of I-bimodules for $U$ such that $\Sigma$, the set of all $\Sigma_{U}$, satisfies (P.0)-(P.4) and such that $\mathscr{P}(U)=$ $\operatorname{ker}(\Sigma, U)$.

Proof. If $U \in V(I)$, set $\Sigma_{U}=\{M \mid M$ is an $I$-bimodule for $U$ such that $[0 \mid M]_{U}^{*} \neq U$ and $\left.\mathscr{P}\left(U /[0 \mid M]_{U}\right)=0\right\}$. Thus, for each $M \in \Sigma_{U}, \mathscr{P}(U) \subseteq$ $[0 \mid M]_{U}$ whence $\mathscr{P}(U) \subseteq \operatorname{ker}(\Sigma, U)$. Since $V(I)$ has $(*), M=U^{*} / \mathscr{P}(U)$ is an $I$-bimodule for $U$ and $[0 \mid M]_{U}=\mathscr{P}(U)$, from which we conclude $\mathscr{P}(U)=\operatorname{ker}(\Sigma, U)$.

Next we prove that $\Sigma$ is a general class of $I$-bimodules. By definition, (P.0) is satisfied. Note also that, since $\mathscr{P}$ is preserved under isomorphisms, if $U, W \in V(I)$ and $U \cong W$, then $\Sigma_{U}=\Sigma_{W}$. From the fact that $\mathscr{P}(U)=$ $\operatorname{ker}(\Sigma, U)$ for all $U \in V(I)$, we have immediately that $U$ is $\Sigma$-semisimple iff it is $\mathscr{P}$-semisimple, and that $U$ is $\Sigma$-radical iff it is $\mathscr{P}$-radical. (Note that $\operatorname{ker}(\Sigma, U)=U$ implies, by (P.0), that $\Sigma_{U}=\varnothing$.) From these observations, (P.3) and (P.4) easily follow. Finally, (P.1) and (P.2) are verified as follows. Suppose $K \triangleleft U \in V(I)$ and $\bar{U}=U / K$. Then $M \in \Sigma_{\bar{V}}$ iff $\mathscr{P}\left(\bar{U} /[0 \mid M]_{\bar{U}}\right)=0$. But $\bar{U} /[0 \mid M]_{\bar{U}} \cong U /[0 \mid M]_{U}$ whence $M \in \Sigma_{U}$. Thus (P.1) is satisfied, and (P.2) is verified similarly. So $\Sigma$ is a general class, as desired.

Remark. If $M \in \Sigma_{U}$, we refer to $M$ as a $\mathscr{P}$-I-bimodule. 
3. Examples and remarks. Examples of general classes of bimodules can be easily obtained. One must seek varieties $V(I)$ which have $(*)$ and examine what radical properties have been studied in them. In this way, Levitzki bimodules can be defined in those varieties studied by Anderson [1] and Zwier [12]. Similarly, prime Jordan bimodules can be defined based upon [11].

As noted at the beginning of the paper, in the variety of associative algebras, the characterization of a radical in terms of modules and bimodules need not coincide (even though one-sided modules form a class of bimodules). For example, the Jacobson radical of an associative algebra can be characterized as the intersection of the annihilators of the irreducible one-sided modules, but, as Sasiada's example of a simple (Jacobson) radical ring illustrates, it need not be equal to the intersection of the annihilators of the irreducible two-sided modules.

The most important observation, however, is that axioms (P.0)-(P.4) can be defined in any algebraic system (in the sense of universal algebra) where there is a meaningful concept of bimodule and birepresentation. If this is the case, most of the above results carry through to this more general system.

Such a system is the class of quadratic Jordan algebras (over $\Phi$ ) as defined by McCrimmon (for information regarding terminology, notation, and basic results in quadratic Jordan algebras, see [6] and [8]). We note that McCrimmon has a satisfactory definition both for bimodule and birepresentation [8]. Thus our theory is seen to carry through as far as Theorem 1, mutatis mutandis. But we also know that if $J=(X, U, 2)$ is a quadratic Jordan algebra, then $J$ can be embedded in a unital quadratic Jordan algebra $J^{\prime}=\left(X^{\prime}, U^{\prime}, 1\right)$. We call $J$ nondegenerate if $\operatorname{ker}\left(U^{\prime}\right)=$ $\left\{x \in X^{\prime} \mid U_{x}^{\prime}=U_{x, y}^{\prime}=0\right.$ for all $\left.y \in X^{\prime}\right\}=0$. We note that if $J$ has no two-torsion, then $J$ is nondegenerate [6, p. 1.32]. If we assume our class of quadratic Jordan algebras is nondegenerate, then our theory carries on through the analogue of Theorem 2.

We can now combine this result with one of Osborn [9] to answer a question posed by McCrimmon in [7] regarding the Jacobson radical and bimodules. In [9], Osborn has given an example showing that the Jacobson radical of a quadratic Jordan algebra need not equal the intersection of the "annihilators" of the irreducible bimodules (in fact, it is not clear whether or not the class of irreducible bimodules form a general class). However, based on the above information, if $J$ is a nondegenerate quadratic Jordan algebra and $R(J)$ is its Jacobson radical, then $R(J)=$ $\bigcap\left\{[0 \mid M]_{J} \mid M\right.$ is a $J$-bimodule such that $[0 \mid M]_{J}^{*} \neq J$ and $\left.R\left(J /[0 \mid M]_{J}\right)=0\right\}$. That is, there is a class of $J$-bimodules such that $R(J)$ can be represented as the intersection of the "annihilators" of members of this class. 


\section{REFERENCES}

1. T. Anderson, The Levitzki radical in varieties of algebras, Math. Ann. 194 (1971), 27-34.

2. V. A. Andrunakievič and Ju. M. Rjabuhin, Modules and radicals, Dokl. Akad. Nauk SSSR 156 (1964), 991-994=Soviet Math. Dokl. 5 (1964), 728-731. MR 32 \#7596.

3. N. J. Divinsky, Rings and radicals, Math. Expositions, no. 14, Univ. of Toronto Press, Toronto, Ont., 1965. MR 33 \#5654.

4. I. R. Hentzel, A note on modules and radicals, Proc. Amer. Math. Soc. 19 (1968), 1385-1386. MR 38 \#4512.

5. N. Jacobson, Structure ar.d representations of Jordan algebras, Amer. Math. Soc. Colloq. Publ., vol. 39, Amer. Math. Soc., Providence, R.I., 1968. MR 40 \#4330.

6. - Lectures on quadratic Jordan algebras, Tata Institute, Bombay, 1970.

7. K. McCrimmon, The radical of a Jordan algebra, Proc. Nat. Acad. Sci. U.S.A. 62 (1969), 671-678. MR 42 \#3137.

8. - Representations of quadratic Jordan algebras, Trans. Amer. Math. Soc. 153 (1971), 279-305. MR 42 \#3139.

9. J. M. Osborn, Representations and radicals of Jordan algebras (to appear).

10. E. Sasiada and P. M. Cohn, An example of a simple radical ring, J. Algebra 5 (1967), 373-377. MR 34 \#2629.

11. C. Tsai, The prime radical in a Jordan ring, Proc. Amer. Math. Soc. 19 (1968), 1171-1175. MR 37 \#6336.

12. P. J. Zwier, Prime ideals in a large class of nonassociative rings, Trans. Amer. Math. Soc. 158 (1971), 257-271. MR 43 \#7478.

Department of Mathematics, Michigan State University, East lansing, MiChIGAN 48823 